\title{
ATRESIA TRICUSPIDEA
}

\author{
Dres. SANTIAGO PRADO, RAFAEL DEL RIO y NEFTALI NAQUIRA
}

\author{
Cátedra de Pediatria del Prof. Audalberto Steeget. \\ Departamento de Cardiología e lnstituto de Anatomía Patoiógica. \\ Hospita! "San Juan de Dios". Santiago.
}

Nos ha parecido de interés dar cuenta de un caso de Atresia Tricúspidea que hemos tenido oportunidad de estudiar y aprovechar en esta oportunidad para hacer una revisión bibliográfica del tema.

\section{CASO CLINICO}

O. C. P. Nació en parto normal de término con $3.050 \mathrm{gr}$, en el Hospital San Juan de Dios el 4 de Septiembre de 1959. La placenta pesó $400 \mathrm{grs}$.

Examinado a las 18 hrs. se le encontró ictérico, por lo que se pidió determinación de grupos sanguíneos de la madre $y$ del niño. Madre: $\mathrm{O} R \mathrm{Rh}+$. Niño: A $\mathrm{Kh}+$; la bilixrubinemia alcanzó a un máximum de $23 \mathrm{mgs} \%$ con $0 \%$ de bilitrubina directa minuto, el día 7-IX-59. Un hemograima de este mismo día muestra poliglobulia de 7.800 .000 g.r. con $158 \%$ de $\mathrm{Hb}$. Leucocitosis de 10.000 . 8 normablastos policromatófilos x 100 leucocitos.

A las 48 hrs, de edad presenta crisis de cianosis, hipotonía y respiración irregular. En estas condiciones se pensó en un daño encefálico. ¿Kernicterus? Se trata con Peristón y Meticortén.

Controlado en el Policlínico de Neurologia en Octubre y Noviembre, se aprecia regresión de los signos neurológicos.

En los días siguientes mejora paulatinamente siendo dado de alta con los diagnósticos de: Enfermedad Hemolitica por incompatibiliad A-O. ¿Daño encefálico?

E1 5-I-60 concurre nuevamente al Policlinico por presentar crisis de cianosis en varias oportunidades, durante ellas retiene la respiración por corto tiempo, se pone morado y cae en sueño para despertar a la 1/2 hora. Hasta ese mamento se alimentaba exclusivamente al pecho. La alimentación se realizaba bien; el chico daba 3 o 4 chupadas y descansaba. Tenía 4 meses de edad y pesaba 5,670 $\mathrm{Kg}$. Lactante activo. Buen panículo. Llanto fuerte. Leve cianosis de los labios y de las extremidades de distribución simétrica. Soplo sistólico del $2^{\circ}$ - $3^{\circ}$ espacio irradiado a los vasos del cuello. Apagamiento del $2^{\circ}$ tono pulmonar. Hígado a 4 cms. del reborde.
Se pide ECG que muestra desviación izqdel eje eléctrico de QRS (Fig. No 1).

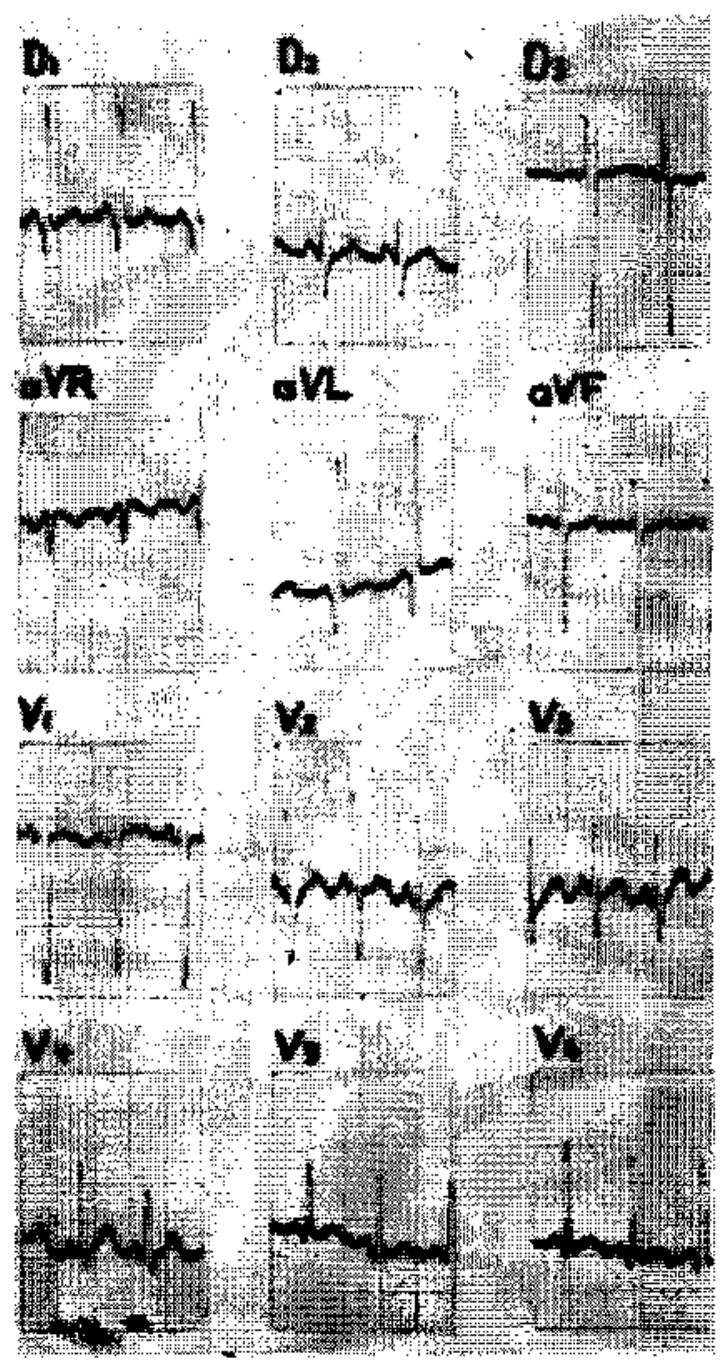

Figura : ${ }^{0}$ 1, E.C.G.: Taquicardia sinusal con frecueacia de $336 \times$ minuto; PR: $0.09 \mathrm{seg} ; \mathrm{AP}$ : $+30^{\circ} ;$ AQRS: - 30\%: P acuminada en D1 ₹ D2: T de escaso yoltaje en DI VL V5 y V6. Conclusión: El aspecto general del trazado hace sospechoso de una Atresia Tricuspidea en presencia del dato clínico de la existencia de cianosis. 
Se pide estudio radiológico (Fjg. 2 y 3 ) donde se aprecia transparenclia aumentada del campo pulmonar, ausencia $2^{\circ}$ arco izq. $y$ falta en VD en lateral izq.

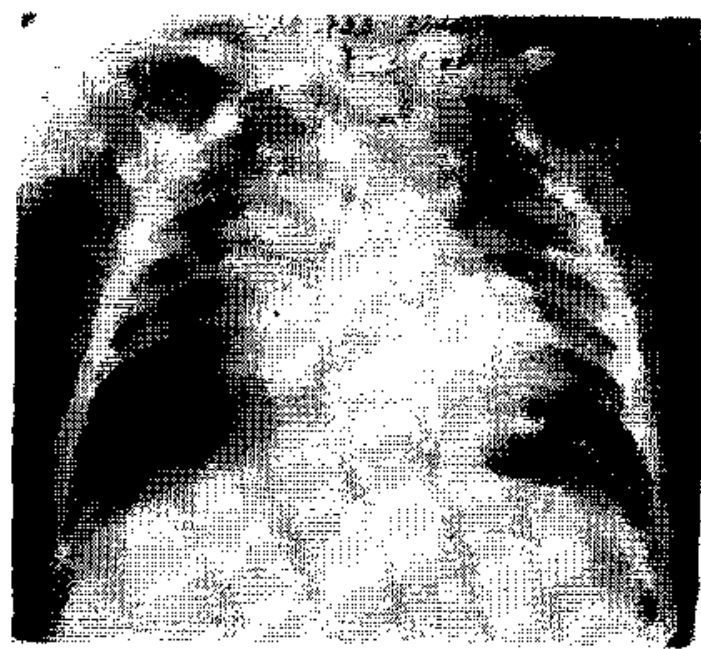

Figura No 2. Postero Anleriot: Campos pulmonares de cransparencia aumentada por acentuada disminu. ción de Ia vascularización.

Corazón: Aumentado de tamaño. Ponta levantada ausencia de $2^{\circ}$ arco izq. Pediculo ensanchado.

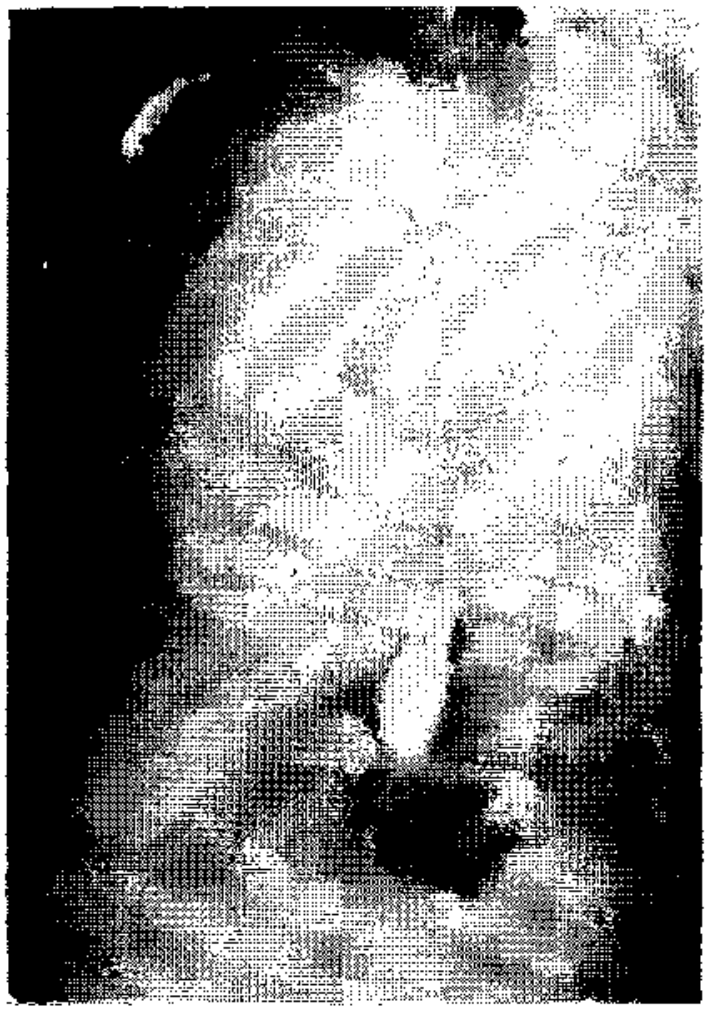

Figura N9 3. Laterat Isquierda: Espacio retroesternal libre por falta de la sombra del VD.
Visto en conjunto con el Depto. de Cardiologia se estima como lo más probable el diagnóstico de: Atresia Tricuspidea. Se cita a control mes a mes.

El 7-II-60 consulta de urgencia par crisis de cianosis. Se encuentra angustiado, cianótico, pulso $200 \mathrm{x}$ minuto. Hígado a $3 \mathrm{cms}$. No si auscultan soplos. Se hospitaliza.

En los días siguientes se agrava, se intensíficó la cianosis, el bígado crece a 5 y 6 cms. lo que se interpneta como insuficiencia cardiaca $y$ se prescribe digital (Cedilanid $y$ Acylanid) y Oxigeno. Posterionmente, hidroclorotiazida. A pesar de todo sigue con mucha disnea $\mathrm{y}$ compromiso general, hasta llegar a la inconciencia. Fallece e 16-II-60. Se Envía a Anatomía Patológica con el diagnóstico de Atresia Tricuspidea, Insuficiencia Cardíaca.

\section{AUTOPSIA}

Lactante. Buen estado pondo estatural. Cianosis moderada de piel y mucosas. Corazón: aumentado de taman̄o, peso 60 grs. (Normal: 30 grs.). Forma alterada por aumento de la aurícula derecha y reducción marcada del ventrículo derecho e hipertrofia y dilatación de las cavidades izquierdas.

Aurícula derecha: con dilatación sacular e hipertrofia de su pared $(2 \mathrm{~mm}$. de espesor). Tricúspide atrésica. Tabique interauricular con orificio oval amplio de 16. $6 \mathrm{mms}$. Desembocadura normal de las venas cavas superior e inferior.

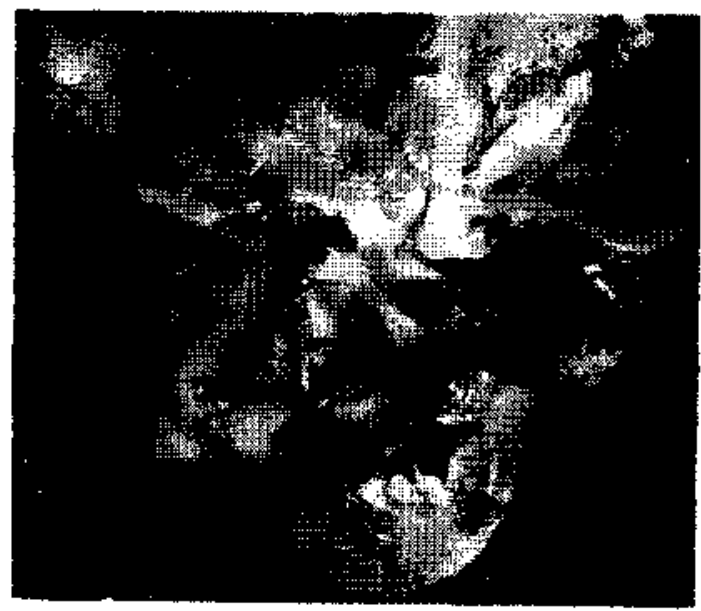

Figura No 4. A. D. hipertrófica y dilatada abierta por su cara superior con el estilete introducido atra. vesando la comunicación interauricular. 
Auricula izquierda: con dilatación mo. derada. Pared de $8 \mathrm{mms}$. de espesor. Desembocadura normal de las venas pulmonares. Mitral normal $(6 \mathrm{~cm}$. de perímetroj. Ventrículo izquierdo: medianamente dilatado, con hipertrofia $19 \mathrm{mms}$. de espesor). Válvula aórtica normal $(3 \mathrm{~cm}$. de perimetro). Comunicación interventricular alta (orificio en hendidura de 3 x 5 mms, ubicado en la pars membranacea).

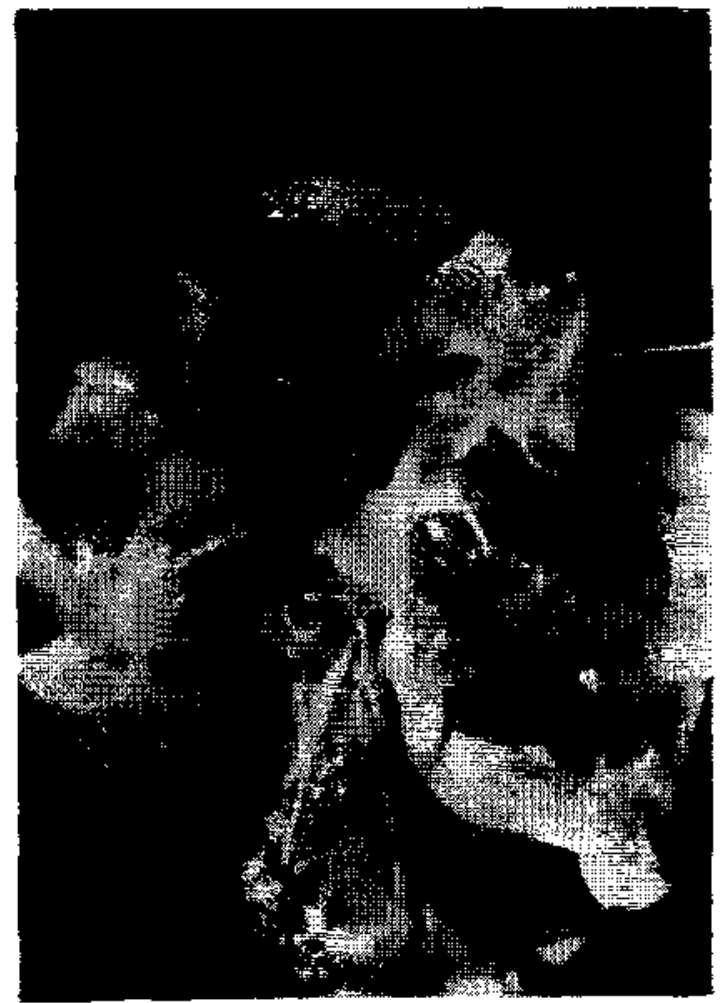

Figura No 5. V.I. Hipettrófico y dilatado, abierto por delante. Comanicación interventricular indicada por el estilete. Aorta y válvula aórtica nomal.

Ventrículo derecho: con cavidad muy reducida (Pared de $6 \mathrm{mms}$. de espesor), a modo de túnel que originándose en la comunicación interventricular, termina en la válcula pulmonar, la cual es normal ( $22 \mathrm{mms}$. de perímetro).

Aorta normal ( $30 \mathrm{mms}$. de perímetro). Coronarias de origen normal. Conducto arterioso cerrado. Arteria pulmonar normal.

Pulmones hiperhémicos con atelectasia de los lóbulos inferiores.

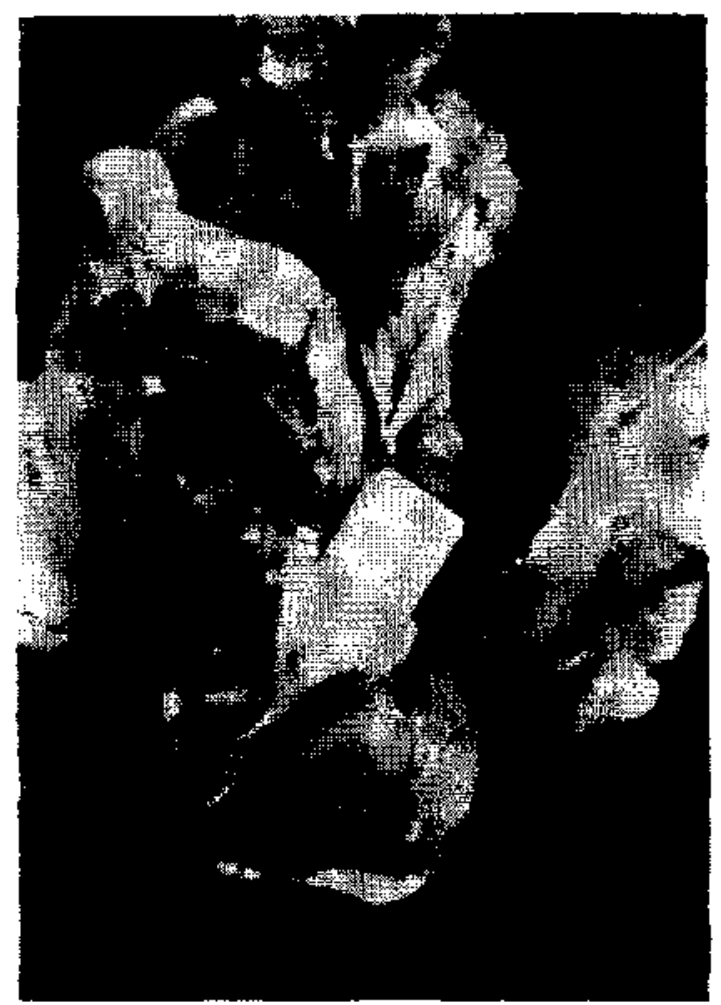

Figura No 6. Cara anterior del corazón. Se ha colocado un estilete qae pasa por el orificio interventricalar hacia \& V.D. hipoplásico y una carculina blanca detrás de la A.P., normal abierta.

Hígado: con aumento moderado de tamaño, hiperhémico y cianótico.

\section{Histología:}

Pulmón: atelectasia y enfisema focal con hiperhemia y pequeñas hemorragias. Sin células cardiacas.

Hígado: atrofia centrolobulillar incipiente.

Las principales características clínicas han sido resumidas ('Edwards ${ }^{8}$ ) en los siguientes hechos:

1. Cianosis progresiva desde el nacimiento.

2. Crisis de anoxia.

3. Soplo sistólico de la base.

4. A $\mathrm{Rx}$ agrandamiento cardíaco.

Configuración que puede parecerse a la Tetralogía de Fallot.

5. E.C.G.: Desviación izq. del eje eléctrico; predominio del ventrículo izq. en precordiales.

Se trata pues de una cardiopatía congénita cianótica cuyo diagnóstico puede ser 
sospechoso fundadamente sobre la base del examen clínico del electrocardiograma y el estudio radiológico.

En esta malformación no existe comunicación directa entre la aurícula derecha y el ventrículo derecho. La sangre de la auricula derecha pasa a la auricula izq. a través de una comunicación interauricular donde se mezcla completamente con Ia sangre arterial que viene por las venas pulmonares.

El curso ulterior de la sangre depende de la serie de malformaciones anatómicas aseciadas y sobre cuya base Edwards y Cols. ${ }^{8}$ las han clasificado en 4 grupos diferentes:

Tipo I: $\sin$ trasposición de los grandes vasos.

a) Con atresia pulmonar y persistencia del Conducto Arteriosos, (Fig. 1) a través del cual pasa la sangre a los pulmones. Forma poco frecuente.

b) Sin trasposición de grandes vasos y con estenosis pulmonar infundibular. En este tipo la sangre llega a los pulmones

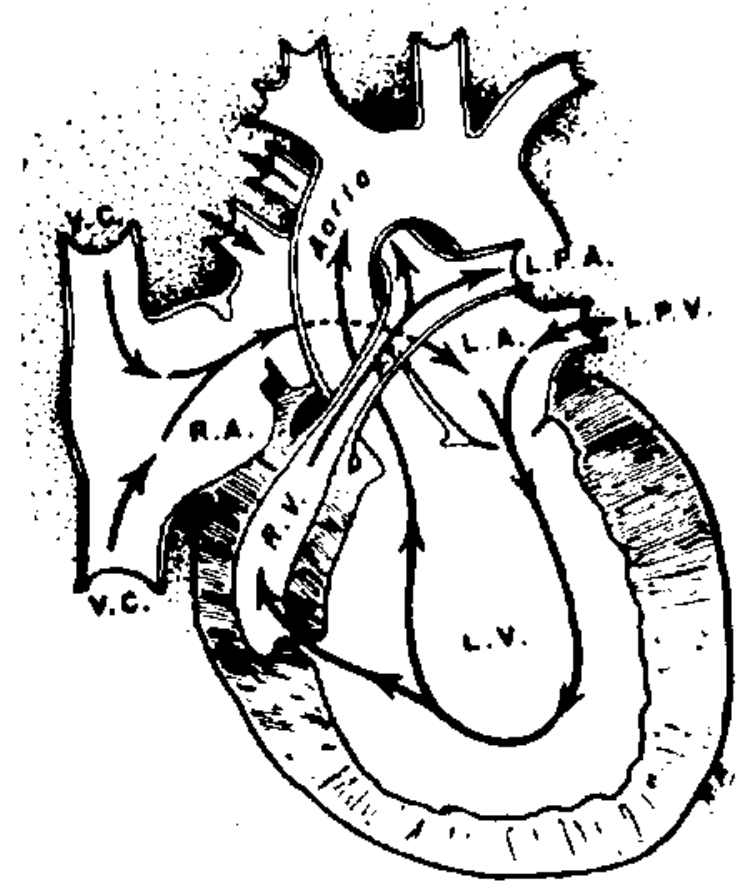

Figura N? 7. Tomado de Edwards 8. a través de un túnel muscular en forma de hendidura, que conecta el ventrículo izquierdo con el derecho que es muy pequeño, a través del cual pasa a la arteria pulmonar.

Se trata de la forma más común y el caso que presentamos corresponde a este tipo.

Tipo II: Con trasposición de los grandes vasos.

a) Con trasposición de los grandes vasos y estenosis pulmonar. La estenosis está en la válvula o lo que es más frecuente, por debajo de ella. La sangre llega a los pulmones a través de la arteria pulmonar y a través de las arterias bronquiales dilatadas.

b) Con trasposición de Ios grandes va5os y $\sin$ estenosis pulmonar.

FISIOLOGía: En la aurícula izq. existe mezcla total de sangre que trae como consecuencia insaturaciónn arterial. El grado de insaturación arterial es consecuencia de la relación entre retorno sistémico y retorno venoso pulmonar. Este a su vez depende del flujo arterial pulmonar ${ }^{13}$.

Siempre existe una comunicación interauricular que es la que permite la mezcla de sangre. La presión en la aurícula derecha es mayor que en la izquierda. Esto trae como consecuencia un aumento de presión venosa sistemica.

cIanosis: Precoz, progresiva, uniforme, intensa ${ }^{9}$. El grado de insaturación arterial y por lo tanto de cianosis depende, como ya comentamos, del flujo pulmonar, de ahí que en los casos con trasposición de grandes vasos $\mathrm{y}$ sin estenosis pulmonar esta puede no estar presente ${ }^{13 \_1 i_{-} 12}$. De los 49 casos citados por Keith ${ }^{11}$ sólo 5 no presentaron cianosis $\mathrm{y}$ todos pertenecían a este tipo. Este mismo autor le asigna gran importancia pronóstica a la precocidad de aparición de la cianosis, concluyendo que en los niños en los cuales ésta es evidente desde el nacimiento el promedio de expectativa de vida es de 5 meses. En los casos en que la cianosis se descubrió después del primer mes de vida, el promedio de edad al morir fué de 4 años y 2 meses. En el caso que presentamos, la cianosis fué leve, precoz y uniforme. 
DEDOS HIPOCRÁTICOS: Es prácticamente constante sobre los dos años de edad. En la serie de Espino Vela estaba presente en 15 de los 19 casos. No estuvo presente en nuestro caso probablemente por su corta edad.

ENCUCLILLAMIENTO: Su frecuencia es discutida, rara para algunos ${ }^{11}$, es común según otros".

DISNEA: Es muy frecuente y acentuada, siendo junto con la intolerancia al ejercicio una manifestación más del cuadro de anoxia. En el niño que comentamos esto se hizo presente, especialmente con el esfuerzo de mamar.

CONGESTIÓN VENOSA $Y$ HEPATOMEGaLIA: La congestión yenosa del cuello ha sido señalada como un signo importante ${ }^{2}$ y para Taussig ${ }^{17}$ la existencia de un higado pulsátil de tamaño normal, combinado con la ausencia de aumento del ventrículo derecho, constituye una presunción diagnóstica de Atresia Tricuspidea con una Comunicación Interauricular relativamente pequeña.

Este signo de la pulsación presistólica hepática es señalado por numerosos autores ${ }^{1{ }_{-}}+1$ y entre nosotros fué consignado en uno de los casos presentados por Duffau ${ }^{7}{ }^{8}$ en cuyo estudio Anátomo Patológico se encontró que la comunicación interauricular correspondia a la mitad de la comunicación aurículo ventricular normal. Nuestro caso presentó congestión venosa $y$ hepatomegalia probablemente como manifestación de insuficiencia cardíaca pero sin latido presistólico.

SEgUNDO TONO PULMONAR: Generalmente es único. Puede presentarse desdoblado en los casos con plétora pulmonar.

soplos: Puede no encontrarse soplos de ninguna especie pero es frecuente que exista un soplo sistólico producido ya sea a nivel de la estenosis pulmonar o a nivel de la comunicación interventricular 1.2 . Espino Vela ${ }^{3}$ encontró que el soplo sistólico era de localización pulmonar en 14 casos y mesocárdico en 5 . En dos de sus casos se presentó soplo diastólico pulmonar. En el caso nuestro encontramos un soplo sistólico suave del foco pulmonar que se hizo difícil de auscultar en las etapas finales.

SIGNOS DE INSUFICLENCIA CARDÍACA: Según la literatura los datos son contradic- torios, para algunos son más frecuentes en los casos con trasposición de grandes vasos y arteria pulmonar normal 11. Relación que no es evidente para otros.

INCAPACIDAD FÍSICA: En general se trata de niños mal desarrollados $y$ nutridos con retraso estático dinámico y pondo es. tatural. A diferencia de esto nuestro niño presentaba buen estado nutritivo $y$ desarrollo estático dinámico normal. En aproximadamente un tercio se asocia a la cianosis, crisis de anoxia seguidas de inconciencia ${ }^{2}$, que fueron muy evidentes y graves en nuestro caso. En contraste con lo anterior existen casos en que la afección es bien tolerada, asi Kyellberg ${ }^{1:}$ señala un caso de 8 años cuya capacidad estaba verdaderamente comprometida.

SOBREvida: Ya señalamos que es pobre, pero existen casos publicados en que es mucho mayor. Duffau " ha dado cuenta de un caso operado a Ios 19 años y en la serie de 16 casos de Abott ${ }^{1}$ se incluye uno que sobrevivió hasta los 56 años.

ELECTROCARDIografía: Eje de $\mathrm{P}$ de $0^{\circ}$ a $\div 40^{\circ}$. La mayoría entre $+20 \mathrm{y}$ $+40^{\circ} 14$. La onda $P$ es alta y bimodal en 15 de los 19 casos de Espino Vela ${ }^{\circ}$. En los casos en que es alta y acuminada, no bimodal, podría explicarse por el reducido tamaño de la C.I.A. ya que ello implicaría una hipertrofia de la A.D. y una pequeña o ausente dilatación de la A.I. ${ }^{14}$. El primer modo estuvo separado del segundo por una distancia no mayor de 0.04 seg. y generalmente fué más alto que éste. En VI fué difásica en todos los casos con predominio de la fase positiva en la mayoría. (11 sobre 19). QRS: Eje eléctrico de $\mathrm{QRS}$ entre $+10 \mathrm{y}-100^{\circ}$ en el plano frontal (Desviación izquierda) en la serie de este mismo autor. Solamerte 3 casos presentaron eje de $\div 35^{\circ}+60^{\circ}$ $y-1-95^{\circ}$.

La desviación izquierda del eje eléctrico constituye la principal clave diagnóstica de esta cardiopatía pero debe recordarse que no es la única cardiopatía congénita cianótica que la produce por cuanto también se ve en el ventrículo único en el atrioventriculario comunis ${ }^{\text {is }}$ en el drenaje anómalo del sistema cava y en el truncus arteriosus persistente ${ }^{13}$, Por otra parte, como ya lo señalamos antes, 
suelen verse casos de Atresia Tricuspídea con desviación derecha del eje eléctrico.

La deflección intrinsicoide está a menudo retardada en precordiales izquierdas $^{3}$ y el total de Jas precordiales son sugestivas de hipertrofia ventricular izquierda con ondas $\mathrm{S}$ profundas en precordiales derechas ${ }^{15}$.

El eje de $\mathrm{T}$ está frecuentemente entre $+60 \mathrm{y}+90^{\circ}$ en el plano frontal.

EXAMEN RADIOLógico: Se trata de un examen de gran importancia diagnóstica pero que puede inducir a error.

POSICIÓN POSTEROANTERIOR: En esta posición puede encontrarse a veces la imagen clásica, en que se ve que el borde derecho no sobrepasa la columna y aparece recto. Punta elevada. Segundo arco izq. cóncavo, campos pulmonares claros.

En otras oportunidades el corazón puede tener el típico aspecto "en sabot". En estos casos es útil buscar un ligero abombamiento de la parte superior del arco inferior izquierdo $y$ que ha sido atribuido a la orejuela izq. dilatada ${ }^{\circ}$. La circulación pulmonar es generalmente escasa pero puede estar aumentada especialmente en 10 casos de trasposición ${ }^{12}$ y puede haber congestión pasiva en los casos de Insuficiencia Cardiaca ${ }^{\circ}$.

En cuanto al tamaño de la sombra cardíaca no todos están de acuerdo y mien. tras para algunos, está siempre aumenta$\mathrm{da}^{13}$, para otros permanence siempre dentro de límites normales ${ }^{11}$. Espino VeIa estima el índice cardiotoráxico de sus casos en la siguiente forma: $+60 \%$ en 3 casos, $+55 \%$ en 5 casos, $+50 \%$ en 15 casos. De esto se deduce que habitualmente está aumentada pero en forma moderada ${ }^{12}$.

O.I.A.: Borde anterior puede ser cóncavo en su parte inferior pero es mucho más frecuente que sea convexo al estar constituído por Ia A.D. Se ha señalado el asincronismo de los latidos entre la parte anterior $\mathrm{y}$ posterior del corazón debido a un aumento en la cavidad sistólica de la aurícula derecha ${ }^{12}$.

O.D.A.: El crecimiento de la aurícula izq. es en general poco importante, aunque se presenta en todos los casos, lo que constituye un signo diferencial con la Tetralogía de Fallot, en la cual se presenta dilatada en raros casos con flujo pulmonar aumentado ${ }^{1 / 2}$.
CATETERISMo CARDíaco: El cateter no logra avanzar desde la A.D. al VOD. y resbala pasando a la A.I. El análisis de los gases muestra gran shunT de der. a izq.

El estudio de curvas de dilución han sido recomendadas en aquellos casos en que se quiere asegurar si existe o nó un pasaje funcional desde la A.D. a la Arteria Pulmonar a través del V.D. ${ }^{4}$.

angIocardografía: Considerado como el examen más útil para el estudio de esta afección. Por medio de él se logran precisar detalles de la A.P. y de sus ramas que son útiles para el cirujano ${ }^{12}$. Ha alcanzado gran desarrollo entre los investigadores suecos.

RECUENTO DE GLóbuLOS ROJOS: Es frecuente la hiperglobulia. En los casos de Duffau 'j todos presentaban recuento de hematíes sobre 6.000.000.

TRATAMIENTo: Todos los autores consultados que se preocupan del tema, recomiendan la operación de Blalock Taussig o de Potts. En Chile, Johow y cols. han dado cuenta de 6 casos operados con estos tipos de intervención, con 2 muertes y mejoría de la cianosis $y$ síntomas cardiacos en el resto. En todo caso estas intervenciones son paliativos destinados a aumentar el flujo sanguíneo pulmonar. Se ha recomendado asociar este tipo de intervenciones a un agrandamiento del tamaño de la C.I.A. con lo que se conseguiría mejorar el débito cardíaco y disminuir la presión en la aurícula derecha. Con este tipo de intervención se habrían mejorado los resultados post operatorios y la sobrevida.

La indicación operatoria se limita a los casos con circulación pulmonar disminuída ${ }^{9}$.

\section{RESUMEN}

Los autores describen un caso de Atresia Tricuspídea en un lactante menor, que fué estudiado clínica y anátomo-patológicamente.

A propósito de él, se hace una revisión de bibliografía sobre este tema, destacándose las principales manifestaciones clínicas de esta afección, su diagnóstico diferencial con otras Cardiopatías Congénitas, el pronóstico y las posibilidades de practicar un tratamiento quirúrgico. 


\section{SUMIMARY}

\section{TRICUSPID ATRESIA}

The authors make a description on a case of Tricuspid Atresia in a young infant, which was studied clinically and pathologically.

On this subject, a review of literature is done, detaching the main clinical symptoms of this condition, its differential diagnosis with other Congenital Malformations of the Heart, the prognosis and possibilities of to perform a surgical treatment.

\section{BIBLIOGRAFĹ}

1.-ABBOT, M. - Citada por Gee. A. L. and Mouteiro. E. 8. Trleuspld Atresfa, British Medicel Journa: November 30: 1283-4, t957.

-ASTLY R. OLDHAM, J. 8 and PARSONS, Canzenital Tricuspid Atresin. Brit. Heart J. 1.5:287, 1958 .

3.-BESOAIN, 3. MANUEL. - Rlectrocardlografia elements1. Editorial Andrég Bello, I959.

4.-BIRKFFAD, C. N. and WOOD, H. E. - The Dlagnosis of Tricuspla Atresta. Proceedings of the Staff Meerlngs of the Mayo Cilnle. 32:506-508, 19.37.

5.-DUFFAU, G.; VIGNAU, A. y MORENO, B. - Considernctones olinlcas sobre 59 casos de Cardlopatias
Congenitas en el nitho operado. Boletir de la Soc. Chilexa de Cardlologie, 2:1-5, 1854.

6.-DUFFAU O., GASTDN, -- Atrebia Trlcuspidea Hipoplasia Ventricular Derecha. Revista Chilena de Pediatria. 266-275, 1950.

7.-DUFFAU, G. - Ventriculo Derecho Hipoplásico $y$ Atresta Trieuspidea. Archivos del Hospltal de NIt́os Roberto deI Río. 1B:38-41, 1950 .

8. - EDWARDS, J. E. and athers. - An Atlas of ConGenital Aromalles of the Heart and Great Vessels. Springfleld, Illinols. Charles C. Thomas, $19 \$ 3$.

9. - ESTINO VELA, J.; BNRKARD, R.; PORTULD, B.; QUIROGA, R. S RUBIO, V. - Atresla Trlcuspidea. Archivos del Instituto de Cardlología de Mexico. $28: 28-62,2938$.

10.-JOHOW, A.: GANTZ. M.; CLFUENTES, A.; NOE, M. y BARTLETT, L. - Experdencla quirúrglea en cardlopatias congénltas en el niño. Boletin de la Sociejad Chllena de Cardlología, 2:6 - 1954.

11, KEITH, JOHN; ROWE, R. D and VLAD P.-Eeart Disease in Infancy and Chilthood. New York. The Macmillan company. 1958.

12.-KJELI-ERRC, SVBN, R.; MANNHEIMER, EDGARD RUDHE ULF, JONSBON BENGT - Diagnosis of Congenltal Heart Disease. Chlcago. The Year Book Publushers Inc., 1955.

13.-NADAS, ALEXANDEk s, - Pediatric Cardilojogy. Phttadelphia and Lomion. W. B. Saunders company, 1957 .

14.--PORTILO, BOLIVAR I ANBELMI. GUILERMO Atresia Tricuspidea. Estudio Electrocardlográfico en 23 casos. Ardh. Inst, Cardio], Méx, 29:341-352, 1959.

15. - SODI-PALARHS, DEMETR.IO RI CALDER, ROYAL M. - - New Basis of Electrocardlogrephy. The C. T. Mosby Compang. 1956 .

16. SoULIE, P, - Cardlopathles Congúnitales. L'EXparsior Sclentitique Francelise, 1956.

17.-TAUSSIG, HELEN B. - Congenitel Malformations of the Heart. The Commonvealth Furd. New York, 1947. 